\title{
Spectrum Sharing in Heterogeneous Wireless Networks: an FP7 CREW use Case.
}

\author{
Stefan Bouckaert ${ }^{1}$, Lieven Tytgat ${ }^{1}$, Sofie Pollin ${ }^{2}$, Peter Van Wesemael $^{2}$, \\ Antoine Dejonghe ${ }^{2}$, Ingrid Moerman ${ }^{1}$, and Piet Demeester ${ }^{1}$ \\ (1) Ghent University - IBBT, \\ Gaston Crommenlaan 8 bus 201 - 9050 Ghent - Belgium. \\ (2) imec, \\ Kapeldreef 75 - 3001 Leuven - Belgium. \\ \{firstname.lastname\}@intec.ugent.be \\ \{firstname.lastname\}@imec.be
}

\section{Introduction}

Cognitive radio (CR) techniques and cognitive networks [1] aim at optimizing the use of the wireless spectrum, by observing the wireless environment and intelligently configuring radio settings and network parameters. The aim of the FP7 CREW project [2] is to establish an open federated test platform in order to facilitate experimental research on advanced spectrum sensing, CR and cognitive networking strategies. The main goal of this demonstration is to showcase the possibilities of the Belgian branch of the CREW federation. A first aspect is the demonstration of the IBBT w-ilab.t testbed [3] which will be incorporated in the CREW federation, through an example CR set-up where Wi-Fi interference is avoided by an IEEE 802.15.4 network using distributed channel selection. Secondly, a high-performance advanced spectrum sensing design [4] by imec, based on reconfigurable analog and digital building blocks is demonstrated, showing the feasibility of spectrum sensing using low-cost low-power handheld devices. Within the CREW project, the integration of the advanced spectrum sensing component and the testbed (i) generates advanced possibilities for executing and monitoring reproducible testbed experiments, and (ii) allows the optimization of horizontal resource sharing between heterogeneous networks.

Blablabla ^ test.

\section{System description and novel characteristics}

(i) Remote systems. Since the IBBT testbed is deployed in an office building in Ghent, Belgium, it is accessed remotely. At each of the 200 nodes' locations, a Tmote Sky device, a routerboard with two Wi-Fi interfaces and an environment emulator (EE) are installed, thus supporting experiments with heterogeneous technologies. The generic integrated analysis and visualization tools ensure fast and accurate data analysis and representation. The custom built EE boards enable advanced logging of test data and physical node characteristics such as power consumption, and allow battery emulation and the manipulation of analog and/or digital input/output pins to emulate events at the sensor nodes. Within the scope of the CREW project, benchmarking strategies are being developed 
that will allow developers to obtain realistic and reliable performance indicators of CR concepts.

(ii) Systems at the demonstration site. An example of the hardware mounted in the testbed will be available during the demonstration. Moreover, the low-power multi-band sensing solution is demonstrated locally. Where most existing platforms target a single technology and are based on laboratory equipment or limited sensitivity off-the-shelf demonstrators, the presented sensing solution is built out of an analog and digital front-end. The analog front-end consists out of the state-of-the-art imec SCALable raDIO (SCALDIO) chip, which is developed in order to support future flexible radios with a tuning range between $100 \mathrm{MHz}$ and $6 \mathrm{GHz}$ and supports cellular, WMAN, WLAN, WPAN and digital broadcast standards. During the demo, two antennas will be connected, allowing to scan the bands from $500 \mathrm{MHz}$ to $2.5 \mathrm{GHz}$, thus covering most relevant bands in the TV, cellular and GSM bands. The digital front-end contains an FPGA to buffer the samples, which are then fed to a PC for analysis.

\section{Features to be demonstrated}

Firmware images containing an interference avoidance scheme are deployed to sensor nodes in the testbed. Based on a local noise scan, each IEEE 802.15.4 node determines on which channel it wants to receive. This channel configuration is visualized in real time using the monitoring functions of the testbed. Next, a $\mathrm{Wi}-\mathrm{Fi}$ access point is activated in the environment of the sensor nodes. In response, the sensor network reconfigures itself to avoid the generated interference, which is again visualized. Then, as an example, the EE is used to emulate a depleting battery. Using the analyzer module, the power consumption data acquired by the EE during the test is visualized. While the sensor nodes reconfigure themselves, this reconfiguration takes approximately 10 seconds due to delays caused by the scanning procedure at the nodes. This is where the imec sensing solution comes into action: assuming 1024 samples are needed at a sampling rate of $40 \mathrm{Msamples} / \mathrm{s}$ for a bandwidth of $20 \mathrm{MHz}$, a $2 \mathrm{GHz}$ band can be sensed in $7.6 \mathrm{~ms}$. To demonstrate this, the scanning procedure is started, which leads to a periodogram, plotting the signal power over time and frequency. For different configuration options, the power consumption of the scanning procedure is estimated. The influence of an access point on the spectrum is demonstrated, and it is indicated how the integration of such scanning functionality in the testbed within the CREW project will enable the development of advanced networking solutions and will provide invaluable information during testbed experiments.

\section{References}

1. Ryan W. Thomas. Cognitive networks. PhD thesis, Blacksburg, VA, USA, 2007.

2. CREW project. Home page. http://www.crew-project.eu/.

3. S. Bouckaert, W. Vandenberghe, B. Jooris, I. Moerman, and P. Demeester. The w-iLab.t testbed. In Proceedings of Tridentcom 2010, Berlin, Germany, May 2010.

4. M. Ingels et al. A $5 \mathrm{~mm}^{2}$ 40nm LP CMOS 0.1-to-3ghz multistandard transceiver. Accepted for ISSCC 2010 . 